\title{
Plasma deposition of constrained layer damping coatings
}

\author{
J A Rongong ${ }^{1 *}$, A A Goruppa ${ }^{2}$, V R Buravalla ${ }^{1}$, G R Tomlinson ${ }^{1}$ and $\mathbf{F}$ R Jones ${ }^{2}$ \\ ${ }^{1}$ Department of Mechanical Engineering, The University of Sheffield, Sheffield, UK \\ ${ }^{2}$ Department of Engineering Materials, The University of Sheffield, Sheffield, UK
}

\begin{abstract}
Plasma techniques are used to generate constrained layer damping (CLD) coatings on metallic substrates. The process involves the deposition of relatively thick, hard ceramic layers on to soft polymeric damping materials while maintaining the integrity of both layers. Reactive plasma sputter-deposition from an aluminium alloy target is used to deposit alumina layers, with Young's modulus in the range $77-220 \mathrm{GPa}$ and thickness up to $335 \mu \mathrm{m}$, on top of a silicone film. This methodology is also used to deposit a $40 \mu \mathrm{m}$ alumina layer on a conventional viscoelastic damping film to produce an integral damping coating. Plasma CLD systems are shown to give at least 50 per cent more damping than equivalent metal-foil-based treatments. Numerical methods for rapid prediction of the performance of such coatings are discussed and validated by comparison with experimental results.
\end{abstract}

Keywords: constrained layer damping, plasma deposition, layered damping coating

\section{NOTATION}

\section{C constant}

$E_{\mathrm{c}} \quad$ Young's modulus of the constraining layer

$f \quad$ natural frequency

$G_{\mathrm{v}} \quad$ shear modulus of the viscoelastic layer

$k_{0} \quad$ rigidity of the system with CLD when $G_{\mathrm{v}}=0$

$k_{\infty} \quad$ rigidity of the system with CLD when $G_{\mathrm{v}}=\infty$

$L \quad$ coverage length

$r \quad=k_{\infty} / k_{0}-1$

$t_{\mathrm{c}} \quad$ thickness of the constraining layer

$t_{\mathrm{v}} \quad$ thickness of the viscoelastic layer

$\eta_{\max } \quad$ maximum modal loss factor

$\eta_{\mathrm{v}} \quad$ loss factor of the viscoelastic layer
FE

PVD

$\mathrm{RF}$

VL finite element physical vapour deposition radio frequency viscoelastic layer

\section{INTRODUCTION}

Constrained layer damping (CLD) is one of the most effective and frequently encountered layered damping treatments $[\mathbf{1 , 2 ]}$. In its simplest form, it comprises a layer of polymeric damping material, typically 50$200 \mu \mathrm{m}$ thick, and a stiff metallic constraining layer (CL), typically $50-200 \mu \mathrm{m}$ thick, on the host structure, as depicted in Fig. 1. The constraining role of the CL is to magnify the dynamic shear deformation in the viscoelastic material, thereby dissipating more energy.

Conventional CLD is available commercially in the form of adhesive metal foils that are applied to the surface of the host structure. A challenge when applying such foils to a structure with a complex surface geometry (e.g. an aerofoil) is to make the stiff CL follow the surface of the host structure exactly. Even slight deviations can induce cleavage stresses across the viscoelastic layer (VL) causing the CL to debond. This can result in the entire system peeling away under external factors such as airflow and foreign object impact.

The MS was received on 7 July 2003 and was accepted after revision for publication on 24 February 2004.

* Corresponding author: Department of Mechanical Engineering, The University of Sheffield, Sir Frederick Mappin Building, Mappin Street, Sheffield S1 3JD, UK. 


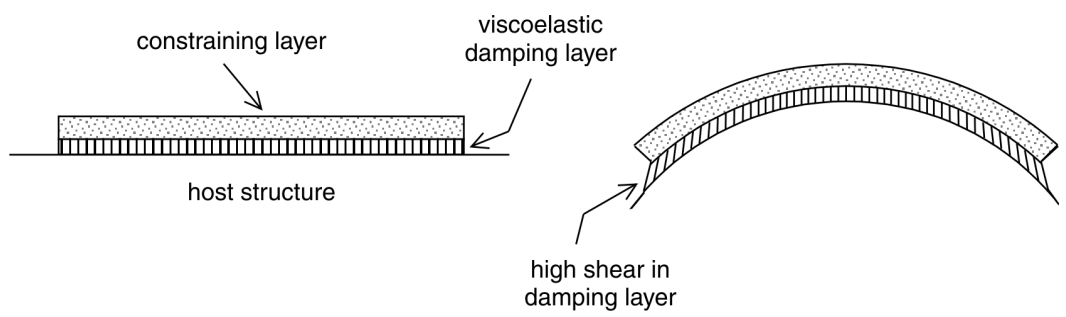

Fig. 1 Schematic of a constrained layer damping system

The most common solution to this problem is to limit the flexural stiffness of the CL; steel CLs applied to complex surfaces are usually no more than $50 \mu \mathrm{m}$ in thickness. Thicker metallic CLs can be pre-formed to the desired shape, however the manufacturing tolerance required on both the $\mathrm{CL}$ and the host increase the fabrication cost significantly. One approach is to make the $\mathrm{CL}$ from a fibre-composite material that is cured after being applied to the surface. Special measures have to be taken, however, to avoid the curing process from chemically altering the properties of the damping material [3].

An alternative approach is described in this paper, namely the use of low-pressure, plasma deposition methods to apply the entire CLD system. Plasma is a gaseous medium consisting of atoms and molecules along with the products of their ionization (ions and electrons), powered by electric or electromagnetic fields. These fields supply energy to a variety of processes in plasma, leading to complex chemical transformations and movements of plasma species. This can be exploited to deposit a wide variety of materials in the form of thin films including metals, polymers [4] and ceramics [5]. With the gaseous nature of the plasma, deposition is possible even on substrates of complex surface geometry, such as cutting tools [6] and turbine blades [7].

The principal challenges in realizing a plasma CLD system are:

(a) the development of a plasma polymer with desired viscoelastic behaviour;

(b) deposition of a thick coating on to a thin, flexible polymeric layer without degrading its damping properties.

While it is possible to circumvent the first challenge by using a hybrid system involving a conventional viscoelastic layer and a plasma CL, the feasibility of the concept depends on a satisfactory deposition method for the hard constraining layer. Prior work in the literature in which hard layers have been deposited on polymeric materials involve only very thin films; alumina films 3$4 \mu \mathrm{m}$ thick have been deposited on $12 \mu \mathrm{m}$ layers of polyethylene terephthalate polymer to act as a gas barrier [8,9]. This paper addresses the development of plasma CLD systems and pays particular attention to the deposition of the relatively thick constraining layer.

\section{SELECTION OF SUITABLE PLASMA METHODS}

The performance of CLD is affected by the properties of both constraining and viscoelastic layers. Different plasma regimes can result in coatings with vastly differing properties. It is therefore necessary to select the desired properties for each layer and the method by which they are created. This section deals with the identification of desired properties and selection of plasma methods to achieve them.

\subsection{Constraining layer}

The maximum damping achievable from CLD depends on the rigidity of the constraining layer and the loss factor of the viscoelastic material. Making the assumption that there is no through-thickness deformation of the viscoelastic material, Marsh and Hale [10] showed that the maximum modal loss factor $\left(\eta_{\max }\right)$ achievable can be approximated using

$$
\begin{aligned}
& \eta_{\max }=\frac{\eta_{\mathrm{v}} r}{2+r+2 \sqrt{\left(1+\eta_{\mathrm{v}}^{2}\right)(r+1)}} \\
& \text { with } r=\frac{k_{\infty}}{k_{0}}-1
\end{aligned}
$$

where $\eta_{\mathrm{v}}$ is the loss factor of the viscoelastic damping material, $k_{0}$ the stiffness of the system when the VL has no shear stiffness and $k_{\infty}$ when the VL is infinitely stiff in shear. The effect of the relative rigidity of the CL to the host structure $(r)$ on the maximum modal loss factor is shown in Fig. 2. It can be seen that for optimum damping it is desirable to make $r$ (and therefore the modulus or thickness of the CL) as large as possible. As added-mass considerations usually limit the size of CL used, a material with a high modulus-weight ratio (specific modulus) would improve performance. The specific moduli of certain ceramics are significantly higher than for metals. Table 1 shows that bulk alumina is around four times higher. For this reason, plasma systems that deposit ceramic layers were considered for the CL deposition phase.

Plasma ceramic films are most often grown by a physical vapour deposition (PVD) method; a sketch to 


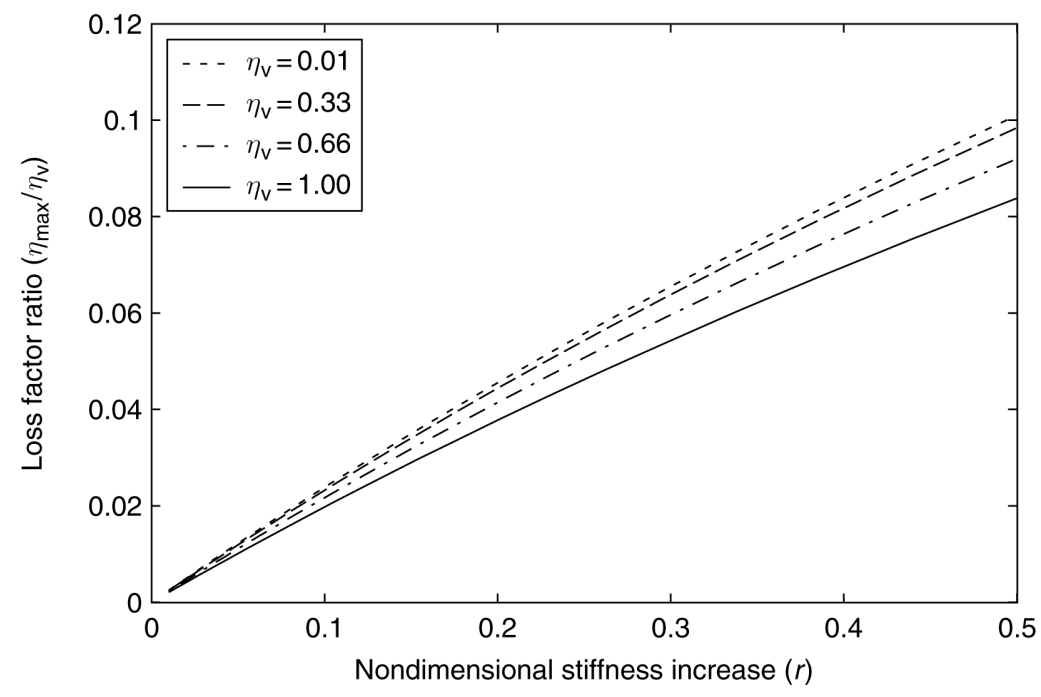

Fig. 2 Effect of parameters $\eta_{\mathrm{v}}$ and $r$ on the loss factor ratio

illustrate the process is provided in Fig. 3a. In PVD, a solid target made of the material to be deposited is present in the plasma. This is sputtered under the effect of energized ions of a neutral gas, such as argon, and redeposited in the form of a thin coating. A variation on this method is reactive PVD, where changes in chemical composition of material occur on the way from a sputter target to the substrate due to the presence of a reactive gas. For example, alumina films can be obtained either via standard PVD, from an alumina target in an argon plasma, or by reactive PVD, from an aluminium target in an argon/oxygen plasma. Radio frequency (RF) plasma sputter deposition has been used to grow alumina coatings up to $200 \mu \mathrm{m}$ in thickness, with values of Young's modulus ranging from 140 to $180 \mathrm{GPa}$ [11]. While this is significantly less than the modulus for bulk alumina, the modulus-density ratio still exceeds that for metals.

\subsection{Viscoelastic layer}

For specifically designed damping materials, $\eta_{\mathrm{v}}$ is typically in the range $0.5-1.5$ over an operating zone of about $50{ }^{\circ} \mathrm{C}$. Shear modulus values usually lie in the

Table 1 Physical properties of various candidate materials for the constraining layer

\begin{tabular}{lclc}
\hline & $\begin{array}{l}\text { Young's } \\
\text { modulus } \\
(\mathrm{GPa})\end{array}$ & $\begin{array}{l}\text { Density } \\
\left(\mathrm{kg} / \mathrm{m}^{3}\right)\end{array}$ & $\begin{array}{l}\text { Specific } \\
\text { modulus } \\
(\mathrm{MN} \mathrm{m} / \mathrm{kg})\end{array}$ \\
\hline Steel & 196 & 7900 & 25 \\
Aluminium & 70 & 2700 & 26 \\
Titanium 6-4 & 115 & 4420 & 26 \\
Alumina (bulk) & 340 & 3400 & 100 \\
Alumina (experiment F) & 220 & 2800 & 79 \\
PZT (Morgan P5) & 80 & 7600 & 11 \\
\hline
\end{tabular}

range $0.1-100 \mathrm{MPa}$. Although equation (1) predicts the maximum achievable damping, it does not say whether it is achievable from a particular configuration. It has been shown [12] that the optimum performance of a thin CLD coating can be achieved when

$$
\frac{G_{\mathrm{v}} L^{2}}{t_{\mathrm{v}} t_{\mathrm{c}} E_{\mathrm{c}}}=C
$$

where $G_{\mathrm{v}}$ and $t_{\mathrm{v}}$ are the shear modulus and thickness of the VL, $E_{\mathrm{c}}$ and $t_{\mathrm{c}}$ are the Young's modulus and thickness of the CL and $L$ is the coverage. The value of $C$ depends on the strain variation over the coated surface [13], for uniform strain $C \approx 10$ rising to $C \approx 50$ for sinusoidal variations [14]. From equation (2) it can be seen that a reduction in the VL achieved by using plasma techniques (say from 50 to $5 \mu \mathrm{m}$ ) would have to be matched by a proportional reduction in shear modulus.

Plasma polymeric films are usually deposited by chemical vapour deposition (CVD) (see Fig. 3b). CVD consists of deposition from a precursor material in gas form, which is chemically activated (ionized or excited) in plasma discharge through collision with electrons, ions or excited atoms and molecules. For example, siloxane plasma polymeric films can be deposited from low-pressure plasma discharge in vapours of HMDSO (hexamethyl disiloxane, $\mathrm{C}_{6} \mathrm{H}_{18} \mathrm{Si}_{2} \mathrm{O}$ ). The deposition of films with Young's modulus ranging from $1 \mathrm{MPa}$ up to several GPa has been reported for HMDSO using RF capacitive discharge [15]. These films also possess good thermal stability, a property important for plasma CLD as the deposition of a ceramic involves considerable energy flux [16], which can alter the polymeric substrate. Coatings deposited from HMDSO exhibit low rates of degradation (below 4 per cent) up to temperatures of $300{ }^{\circ} \mathrm{C}$ due to the strong $\mathrm{Si}-\mathrm{O}$ bonds [17]. 


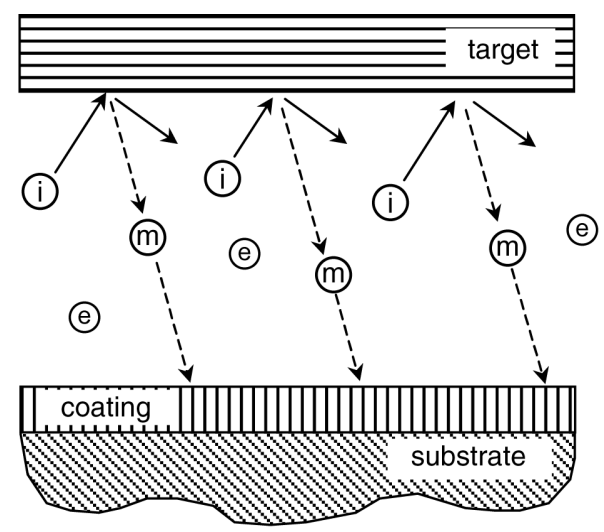

(a) Physical vapour deposition
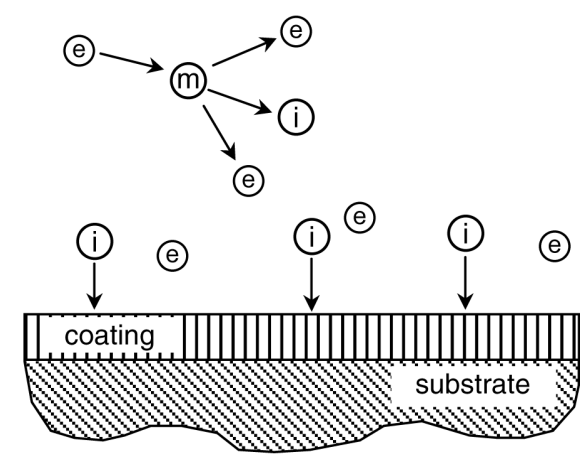

(b) Chemical vapour deposition

Fig. 3 Vapour deposition techniques ((m) molecule, (1) ion and (e) electron)

Accurate measurements of the loss factor of such coatings, however, have not yet been made. In order to assess the performance of the novel CL described in the previous section it was essential to have a reliable measure of the loss factor of the polymeric material. Accordingly, several hybrid systems (plasma CL on a conventional damping polymer) were studied. One single material satisfying thermal stability, low modulus and high loss factor was not found. Instead, deposition of ceramic layers was attempted on viscoelastic layers made from one material satisfying the thermal stability requirement - the silicone rubber, Sylgard 184, from Dow Corning [18] - and one with suitable modulus and damping properties - the acrylic-based ISD112 from $3 \mathrm{M}[19]$.

Initial studies depositing HMDSO yielded a coating $10 \mu \mathrm{m}$ in thickness with a modulus of approximately $5 \mathrm{GPa}$. While it was relatively easy to deposit a hard layer over this, it was not successful as a damper because the material was far from its glass transition (peak $\eta_{\mathrm{v}}$ ) and the shear stiffness was too high for most practical applications; equation (2) indicates an optimum coverage length of around $0.5 \mathrm{~mm}$. Rather than attempting to improve the plasma-deposited polymeric layer, efforts were instead focused on achieving a suitable CL-the more fundamental challenge. Thus, further development of the fully plasma CLD was suspended and hybrid systems were studied.

\section{EXPERIMENTAL COATING DEPOSITION}

\subsection{Development of deposition methodology}

Reactive sputter deposition from an aluminium target was selected as the most appropriate method for growing thick (up to $300 \mu \mathrm{m}$ ) alumina films, as it is a well-understood and established process. By varying the gas composition, e.g. the content of oxygen in argon, it is possible to deposit alumina-like coatings of variable stochiometry [20]. This approach facilitates control of internal stresses in the film, crucial for the integrity of thick coatings.

Initial experiments were conducted using an RF planar magnetron, based on a conventional RF capacitive discharge configuration. The system was converted to an RF magnetron (see Fig. 4) by placing stacks of permanent magnets under the powered electrode. The aluminium target, placed on the powered electrode $70 \mathrm{~mm}$ from the substrate, formed a thin surface alumina layer when oxygen was added to the gas mixture, thereby providing a source of raw alumina, which was sputtered by energetic ions from plasma. A significant part of the ion energy went into thermal heating of the target and thence the entire interior of the vacuum chamber. In order to reduce the heating of the sample, the substrate was cooled with circulated water. Nevertheless, in the RF magnetron reactor only siloxane

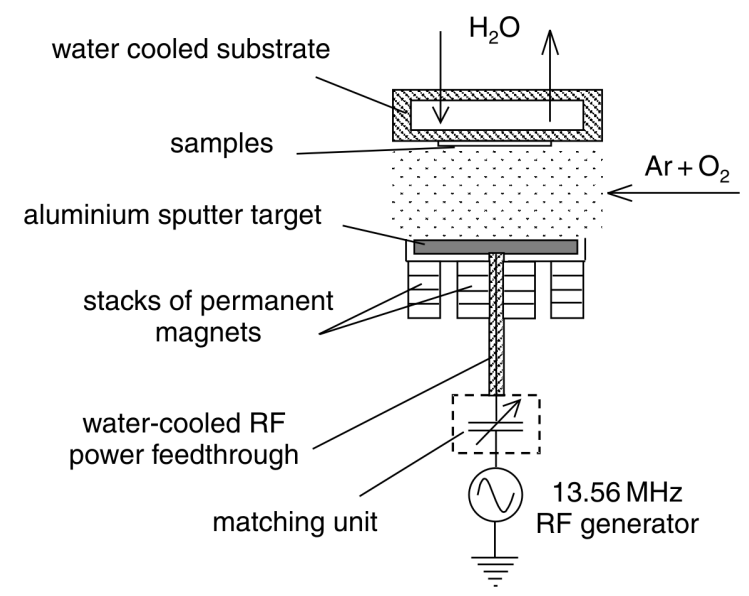

Fig. 4 RF planar magnetron used to deposit alumina coatings 


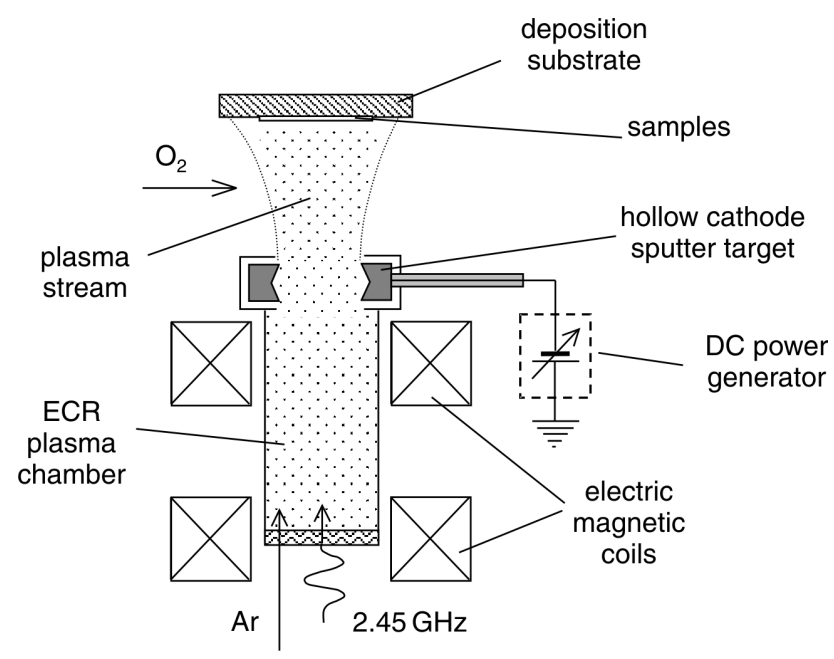

Fig. 5 ECR-hollow cathode arrangement for alumina coating deposition

polymers withstood deposition of ceramic coatings without degradation. Polymer materials of lower thermal stability disintegrated, affected by a combination of ionic and thermal fluxes despite water cooling of the substrate.

To reduce the heat load of the samples and increase deposition rates, a novel sputter deposition configuration based on the electron cyclotron resonance (ECR) reactor was developed (see Fig. 5). The idea of using ECR plasma reactor for sputter deposition purposes dates back to the work of Ono et al. [21]. The novelty of the current configuration is a hollow cathode circular target that radiates little heat as it does not face the substrate and has direct water cooling. With this system, although the applied power was increased (from the RF magnetron) by a factor of 10 (see Table 2) the temperature of the substrate $(100 \mathrm{~mm}$ away) remained below $60{ }^{\circ} \mathrm{C}$. At the same time, the plasma stream directed material, sputtered from the target, towards the samples. The modifications resulted in increased deposi- tion rates: the ECR hollow cathode reactor was typically 5-7 times quicker (see Table 2).

\subsection{Characterization of ceramic deposits}

Ceramic layers were simultaneously deposited on beam specimens with and without polymer films. Those without polymer films were used to characterize the coating material. Numerous experiments were carried out using different parameter combinations. The results of six successful experiments are summarized in Table 2.

The thickness of alumina coatings was measured using a digital micrometer with a resolution of $1 \mu \mathrm{m}$ by subtraction of the beam thickness from the net thickness of the beam and coating. The density of the coating for configuration $\mathrm{F}$ was also obtained by measuring the beam mass (with a resolution of 0.01 grams) and relating that to the change in volume. It is interesting to note that the density obtained for the coating is considerably lower than that for the bulk ceramic (see Table 1).

The hard coating Young's modulus was measured by a nanoindentation method [22] using a Hysitron Triboscope coupled with a Digital Instrument Dimension 3100 (Veeco) atomic force microscope (AFM). This apparatus configuration combines the benefits of high positioning accuracy and imaging capabilities of AFM with automation and the small indentation forces of the nanoindentor. Indentations were made using a probe with Berkovich tip geometry. Typical indent loads were $500-2000 \mu \mathrm{N}$, resulting in probe penetration depths up to $100 \mathrm{~nm}$. The gradient of the unloading curve was used to obtain the indentation modulus; note that the Poisson's ratio of the coating was taken to be 0.22 , the same as that of recrystallized (bulk) alumina. The Young's modulus of the coating was assumed identical to the indentation modulus for analytical validation studies.

The coating developed in experiment $\mathrm{F}\left(E_{\mathrm{c}}=\right.$ $220 \mathrm{GPa}$ ) was considered the best for plasma CLD studies, both in terms of modulus, thickness and

Table 2 Deposition parameters and properties of alumina coatings

\begin{tabular}{llllllll}
\hline & & \multicolumn{6}{c}{ Configuration } \\
\cline { 3 - 8 } Parameter & Units & A & B & C & D & E & F \\
\hline Reactor & & \multicolumn{2}{c}{ RF } & magnetron & \multicolumn{5}{c}{ ECR-hollow cathode } \\
Concentration of $\mathrm{O}_{2}$ & $\%$ & 5 & 5 & 13.3 & 14.5 & 12 & $12-14$ \\
Pressure & $\mathrm{m}$ torr & 10 & 10 & 3.6 & 10 & 10 & 15 \\
Power & $\mathrm{W}$ & 200 & 300 & 4000 & 4000 & 4000 & 4000 \\
Deposition time & hours & 120 & 180 & 1 & 3 & 2.9 & 13 \\
Deposition rate & $\mu \mathrm{m} / \mathrm{h}$ & 0.45 & 1.86 & 6.1 & 13 & 5.9 & 4.6 \\
Coating properties & & & & & & & \\
$\quad$ Modulus & $\mathrm{GPa}$ & 94.9 & 77 & 157 & 197 & 122 & 220 \\
$\quad$ Thickness & $\mu \mathrm{m}$ & 54 & 335 & 6.1 & 39 & 17 & 60 \\
\hline
\end{tabular}


deposition rate and for durability and minimal damage to the viscoelastic material.

\subsection{Polymer substrate: materials and preparation}

For CL deposition studies, Sylgard 184 was used because of its thermal stability up to $300{ }^{\circ} \mathrm{C}$. High levels of damping were not expected, as the glass transition is around $-120{ }^{\circ} \mathrm{C}$. However, its low Young's modulus (approximately $5 \mathrm{MPa}[\mathbf{1 8}]$ ) was considered to provide a good test of the deposition method.

A $25 \mu \mathrm{m}$ thick Sylgard 184 film was applied using a doctor blade on a titanium strip (dimensions $120 \mathrm{~mm} \times 12 \mathrm{~mm} \times 0.95 \mathrm{~mm}$ ). To ensure good adhesion between the ceramic and the polymer layer, the surface of the polymer was exposed for 5 minutes to oxygen plasma which cleaned and oxidized the polymer surface, promoting bonding of the overlaying alumina film. Using the RF magnetron technique described above, a $335 \mu \mathrm{m}$ thick alumina layer was successfully deposited on top of the Sylgard film.

The work was then extended to deposit alumina coatings on to a real damping material. For this purpose, alumina was deposited on to a polymer with known damping properties: the popular ISD 112 selfadhesive tape widely used in conventional CLD applications. A layer, $100 \mathrm{~mm}$ in length and initially $50 \mu \mathrm{m}$ in thickness, was pressed on to a degreased steel beam (dimensions $130 \mathrm{~mm} \times 12 \mathrm{~mm} \times 0.5 \mathrm{~mm}$ ) to give a coating approximately $40 \mu \mathrm{m}$ in thickness. Again, a 5 minute pre-treatment in oxygen plasma was used to activate the surface. Finally, the optimized ceramic layer (configuration F) was deposited over the viscoelastic layer to give a CLD coating extending approximately $100 \mathrm{~mm}$ from one end of the beam. In total, four nominally identical specimens were produced in this way. One anomaly noted was that although $60 \mu \mathrm{m}$ ceramic coatings were deposited on the plain steel beams, the thickness increase of beams already coated with a layer of ISD112 was only $30-40 \mu \mathrm{m}$.

\section{ANALYTICAL METHODS}

Changes in natural frequency and modal loss factor are the most common ways of quantifying the effect of a CLD system on a structure. For real components, particularly those with complex geometry, finite element (FE) analysis is the most popular way to predict modal properties. For a system with CLD, the main departures from a standard, linear modal analysis is that the modulus of the viscoelastic layer varies with frequency and is a complex number. While the effectiveness of internal variable methods such as that of Golla, Hughes and McTavish (GHM) [23] has been demonstrated [24], such approaches are not widely used, as they are currently not included in commercial FE software. Instead, modeby-mode iteration [25] can be used to allow for frequency dependence. As few FE packages allow eigenvalue analyses with a complex modulus, approximate methods based on real eigenvalue analyses have been developed. The simplest of these are the standard modal strain energy (MSE) method [26] and a recent modification [27].

Simple methods are highly desirable for design studies to minimize computation effort, as application of CLD to an FE model increases its size considerably; this is exacerbated by that fact that CLD systems are very thin as a relatively high mesh density is required to retain acceptable element aspect ratios (typically $<100$ ). The approximate method for predicting maximum achievable damping developed by Marsh and Hale [10] is a useful tool in the design of CLD for real components. While the accuracy of the method has previously been demonstrated by comparison with experimental data [10], to the authors' knowledge, a direct comparison with other numerical methods has not been made. A brief study is presented here to demonstrate the accuracy of the method [i.e. the validity of equation (1)] by comparing it with more traditional, numerical approaches.

A cantilever beam fully covered with CLD was used for this study (properties are given in Table 3). Four existing methods were used to calculate the sensitivity of the natural frequency and loss factor of the first five modes of the beam to the shear modulus of the VL. Two of the methods involved complex numbers: one was a Rayleigh-Ritz solution of the analytical model presented by Miles and Reinhall [28] and the other used the specialist finite element code FINES [29]. The other two predictions were obtained using the more common standard and modified MSE methods. Results for the second mode (for the modulus range considered, the second mode shows the loss factor curve fully) are presented in Fig. 6. In all the cases studied, the two methods using complex numbers gave identical results. It can be seen in Fig. 6 that the standard MSE significantly overpredicts the damping while the modified version gives a slight underprediction. Note that loss factor curves for other modes were similar in shape to those presented: maximum levels were identical but

Table 3 Properties of the beam used for numerical study

\begin{tabular}{llll}
\hline Part & Property & Units & Value \\
\hline Base beam & Length & $\mathrm{mm}$ & 100 \\
& Thickness & $\mathrm{mm}$ & 0.5 \\
& Young's modulus & $\mathrm{GPa}$ & 196 \\
& Density & $\mathrm{kg} / \mathrm{m}^{3}$ & 7900 \\
Viscoelastic layer & Thickness & $\mu \mathrm{m}$ & 50 \\
& Density & $\mathrm{kg} / \mathrm{m}^{3}$ & 1000 \\
& Loss factor & - & 1 \\
Constraining layer & Thickness & $\mu \mathrm{m}$ & 40 \\
& Young's modulus & $\mathrm{GPa}$ & 220 \\
& Density & $\mathrm{kg} / \mathrm{m}^{3}$ & 2800 \\
\hline
\end{tabular}




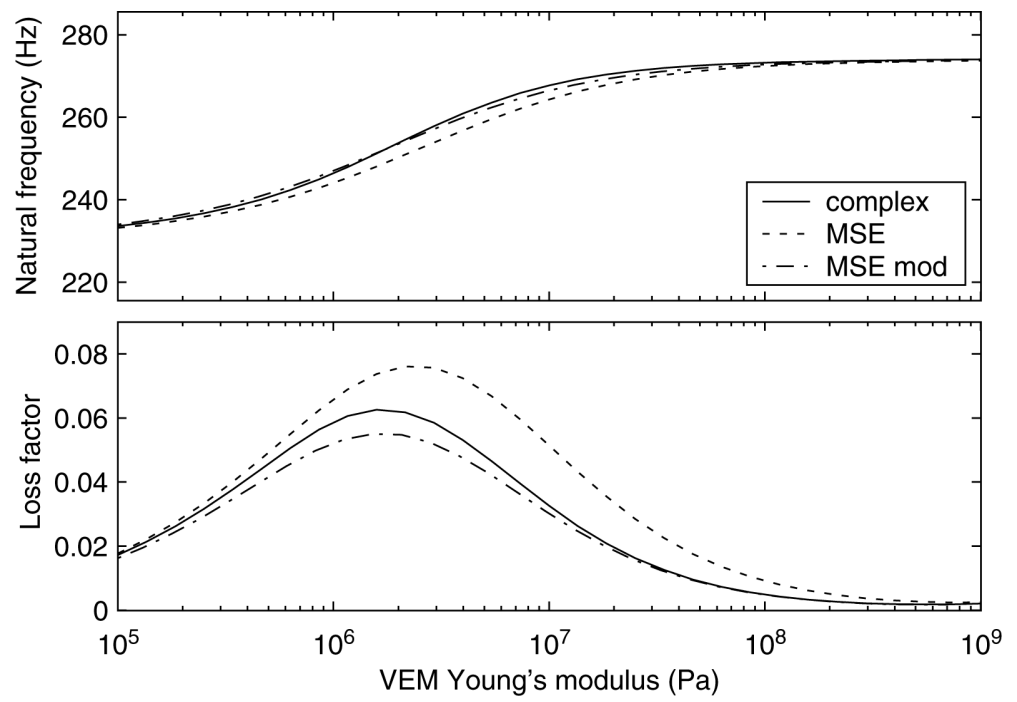

Fig. 6 Comparison of calculation methods on the cantilever CLD example (mode 2) (VEM, viscoelastic material)

occurred at different values of shear modulus $(0.2 \mathrm{MPa}$ for the first mode and $4 \mathrm{MPa}$ for the third).

The Marsh and Hale (MH) method relies on the correct identification of stiffness parameters $k_{0}$ and $k_{\infty}$. For a simple structure such as beams, closed-form expressions for these parameters have been obtained based on the classical Ross, Kerwin and Ungar (RKU) analysis $[11,30]$. In the more general case, however, where FE models are used, the modal stiffness (i.e. modal mass $x$ frequency $^{2}$ ) has to be used. It was found that for relatively thin coatings (less than a fifth of the thickness of the host structure) $k_{0}$, the modal stiffness of the coated beam when the VL carries no shear stress, was almost identical to the stiffness of the plain structure. The modal stiffness of the coated beam with infinite shear stiffness of the viscoelastic layer $\left(k_{\infty}\right)$ was identified for models with three different levels of complexity. In each case the CLD system with the VL infinitely stiff in shear was approximated using a layer of elements with:

(a) properties of the CL applied directly to the surface of the host structure;

(b) modulus and density of the CL but with the combined thickness of both layers applied directly to the surface of the host structure;

(c) properties of the CL, offset from the surface of the host structure by the thickness of the VL and constrained to allow no shear across the gap.

A comparison of peak loss factors predicted using MH methods with those from standard analyses is given in Table 4. For this comparison, the FE prediction using complex numbers was assumed to give the 'exact' solution. The table shows that the $\mathrm{MH}$ method can give good approximations of peak damping (better than other approximate methods), but it is important to model the CL offset accurately (compare method 3 against methods 1 and 2).

\section{ANALYSIS AND TESTING OF PLASMA CLD SPECIMENS}

Modal properties (natural frequency and loss factor) of specimens with plasma CLD coatings were measured and compared with predictions obtained using the methods described in the previous section.

\subsection{Room temperature evaluation}

Two different experimental arrangements were used. A cantilever arrangement, in which the uncoated part of the beam was clamped securely in a heavy block, was used to obtain accurate measurements at room temperature. A sketch of the apparatus is presented in Fig. 7. Random excitation was provided by means of a magnetic transducer $15 \mathrm{~mm}$ from the clamp. A laser displacement probe was used to measure the response near the tip. Note that the exciter and sensor were positioned carefully along the beam centre-line to obtain results for flexure modes only. The frequency response

Table 4 Comparison of the maximum loss factor of the cantilever beam using different methods

\begin{tabular}{llcr}
\hline Approach & Method & $\eta_{\max }$ & \% error \\
\hline Rayleigh-Ritz & Miles and Reinhall [28] & 0.0660 & 0 \\
Full FE model & Complex eigenvalue & 0.0660 & 0 \\
& MSE & 0.0799 & 21.2 \\
Analytical model & MSE modified & 0.0579 & -12.2 \\
Marsh and Hale [10] & RKU analysis & 0.0702 & 6.4 \\
& $\begin{array}{l}\text { Modal properties } \\
\text { from FE (method 1) }\end{array}$ & 0.0527 & -20.3 \\
& $\begin{array}{l}\text { Modal properties } \\
\text { from FE (method 2) }\end{array}$ & 0.0965 & 46.3 \\
& Modal properties & 0.0708 & 7.3 \\
& from FE (method 3) & & \\
\hline
\end{tabular}


CLAMPED-FREE

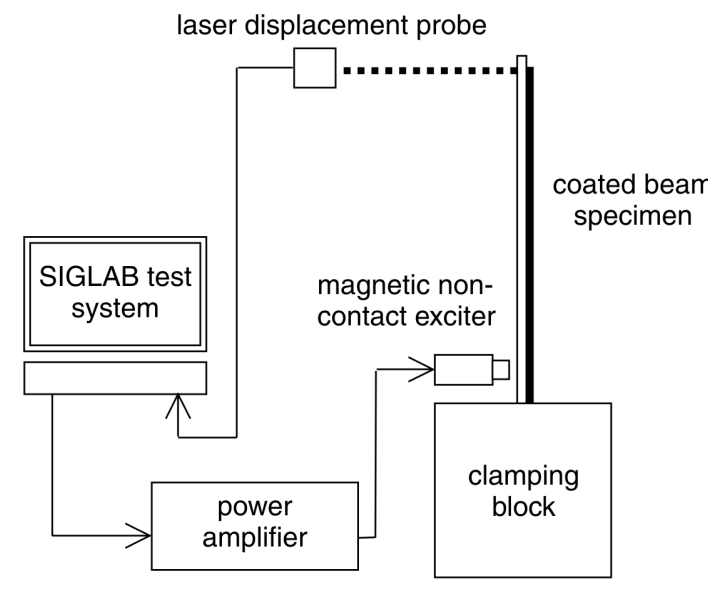

FREE-FREE

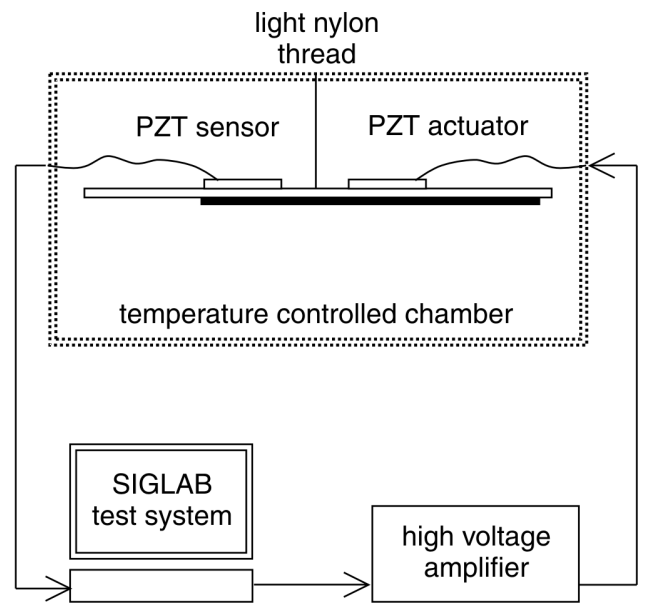

Fig. 7 Vibration test configurations used to obtain modal properties

function (displacement over excitation voltage), averaged over 100 records, was obtained using a Siglab ${ }^{\mathrm{TM}}$ data acquisition system. Modal properties were then obtained using a modified version of the circle-fit method.

A beam with no coatings was first tested in this setup to identify background damping levels. The average modal loss factor over the first five modes was found to be around 0.001 , with no mode being higher than 0.002 . The natural frequencies and measured mass density were used to obtain the Young's modulus of the steel substrate material $(196 \mathrm{GPa})$. The coated specimens were then tested in the same way at an ambient temperature of $22{ }^{\circ} \mathrm{C}$. The specimen-to-specimen standard deviation in natural frequency was 0.4 and 22 per cent for the modal loss factor. Averaged results for the first three modes are compared with those predicted using the complex FE method in Fig. 8. In this example, the nodes related to the CL in the FE mesh were not restrained at the root in order to match the experimental configuration and the prediction was carried out using frequency-dependent complex modulus properties.

From Fig. 8 it can be seen that the addition of the coating increased natural frequencies in all modes. There is good agreement between predicted and measured values, although the prediction overestimated natural frequencies by up to 2 per cent. Modal loss factors of approximately 0.03 were predicted and measured for each mode. Differences between measured and predicted results are of a similar order to the specimen-to-specimen variation.

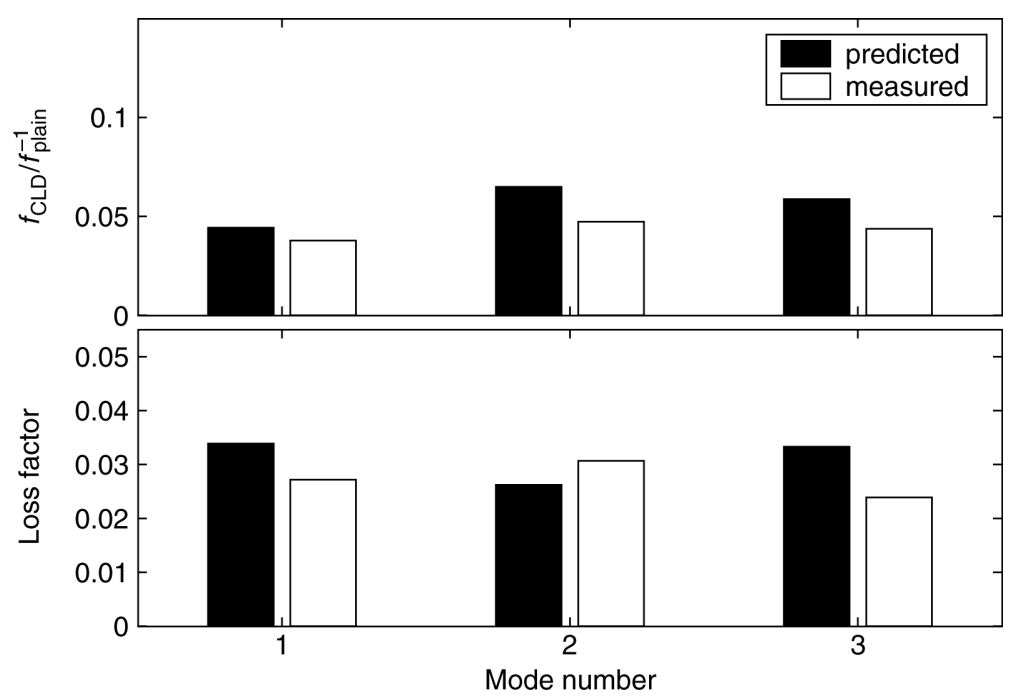

Fig. 8 Predicted and measured modal properties (clamped-free test) 


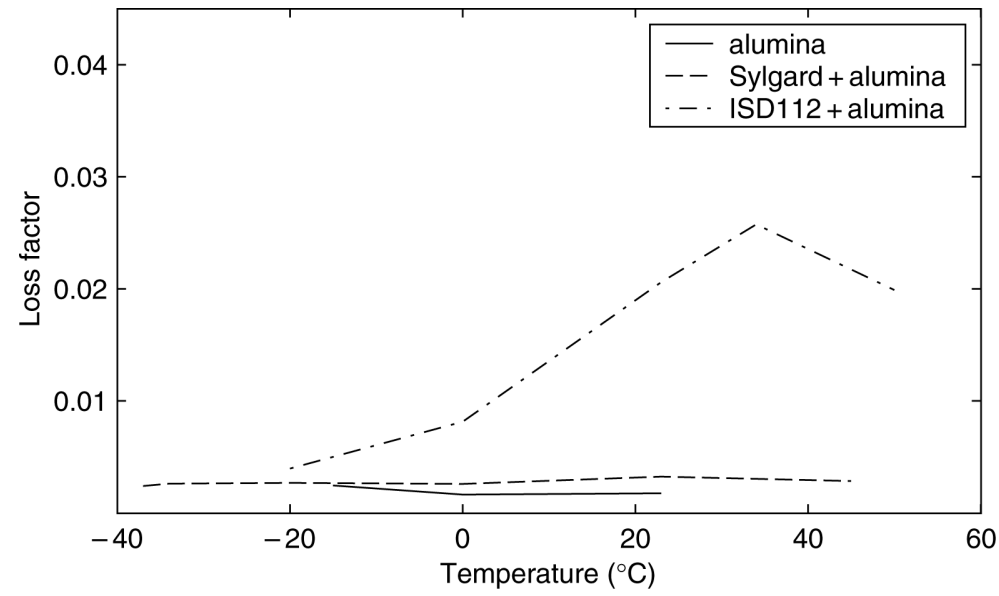

Fig. 9 Measured modal loss factors for freely suspended beams with various coatings

\subsection{Effect of temperature on performance}

A second series of tests was carried out in a temperature-controlled chamber to assess the performance of the CLD coating over a wide temperature range. For this study, the test apparatus had to be altered slightly as it was not possible to get the laser head into the temperature-controlled chamber (see Fig. 7). Instead, excitation and measurement were achieved using small piezoelectric transducers (PZTs) $(12 \mathrm{~mm} \times 3 \mathrm{~mm} \times 0.3 \mathrm{~mm}$ patches of Mogran P5 ceramic). The beam was suspended on a light nylon thread to simulate free boundary conditions. A drive signal of around $100 \mathrm{~V}$ peak-to-peak was found to be ample for the tests carried out. In this case, the frequency response was the voltage measured by the sensor PZT divided by the driving voltage. Again, modal properties were obtained by curve fitting.

One CLD coated beam with ISD112 was tested over the range -20 to $50{ }^{\circ} \mathrm{C}$. As a comparison, a CLD system with Sylgard 184 and a beam simply coated with a ceramic layer were also tested. Averaged results (the standard deviation of results was around 20 per cent) for the first six flexure modes are presented in Fig. 9. The Sylgard and plain ceramic specimens show that the background damping from this approach is less than 0.002 . The ISD112-based system, however, shows a variation in loss factor, rising from near background levels at $-20{ }^{\circ} \mathrm{C}$ to a maximum around 0.03 at $35{ }^{\circ} \mathrm{C}$. This characteristic follows those of the ISD112 material showing that the CLD mechanism is working and that deposition of the hard layer has not destroyed the ability of the viscoelastic material to dissipate energy.

Predictions were also carried out for this arrangement. In this case, extra elements were added to the FE mesh to model the PZT sensors (see Table 1 for properties). Results for the best (mode 1) and worst (mode 3) matches between measured and predicted values are presented in Fig. 10. In general, the variation of natural frequency with temperature is predicted accurately. Predicted values of loss factor, however, are generally higher than those measured. This is
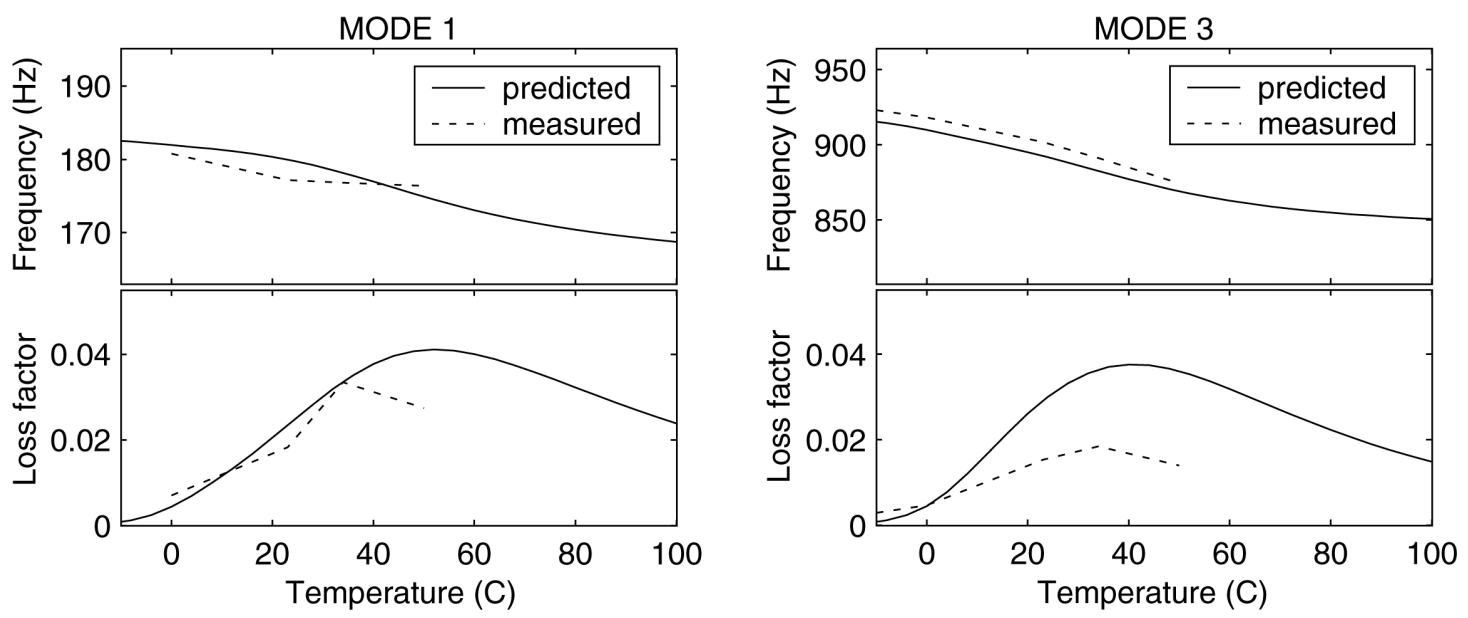

Fig. 10 Predicted and measured modal properties of a freely suspended beam with ceramic coating over ISD112 
particularly noticeable above $40{ }^{\circ} \mathrm{C}$ in mode 1 and above $20{ }^{\circ} \mathrm{C}$ in mode 3 .

One explanation of the difference between measured and predicted results is that some of the damping performance of the VL has been lost. This is supported by the earlier observed fact that the overall thickness of the CLD specimens after coating was less than expected; molecules building up the hard layer may have penetrated into the damping material, modifying its properties slightly. For the specimens and modes considered, this reflects a $10-40$ per cent reduction in peak loss factor of the viscoelastic material $\left(\eta_{\mathrm{v}}\right)$.

\subsection{Predicted performance}

The effectiveness of the new damping coating developed is demonstrated numerically on an axial compressor blade. The prediction was carried out using Marsh and Hale's method but was compared with a full solution for several combinations and found to have similar accuracy to the cantilever study presented in section 4 .

Figure 11 shows the maximum damping that could be achieved for the first mode of such a blade, with a plasma CLD coating with properties similar to the one developed $\quad\left(E_{\mathrm{c}}=220 \mathrm{GPa}, \quad \rho_{\mathrm{c}}=2800 \mathrm{~kg} / \mathrm{m}^{3} \quad\right.$ and $\eta_{\mathrm{v}} \approx 0.8$ ). A modal loss factor of 2 per cent is usually considered excellent for this type of structure. With plasma CLD, it can be seen that this could be achieved with a coating less than $100 \mu \mathrm{m}$ in thickness.

The damping benefits of the plasma ceramic layer over conventional metal foils can be illustrated by comparing the relative damping achieved by each method in this configuration. Figure 12 shows the maximum modal loss factor obtained against the total treatment thickness and the relative mass increase. Here, the VL thickness is $30 \mu \mathrm{m}$, representing the typical minimum limit for conventionally applied tapes. In this study, the plasma process is assumed to cause a 20 per cent reduction in the loss factor of the viscoelastic material while no reduction is assumed for the traditional treatments. It can be seen that the thickness (and hence volume) of plasma-deposited ceramic required to achieve a given level of damping is similar to the best conventional material (steel). The mass penalty, however, is significantly reduced: e.g. the overall mass increase to achieve a modal loss factor of 2 per cent is around 0.5 per cent for plasma CLD rather than 1 per cent for both traditional CLD treatments.

\section{CONCLUSIONS AND FURTHER WORK}

In this paper, a novel damping coating, namely plasma CLD, has been described and demonstrated. Ceramic coatings have been deposited successfully on soft polymeric materials using RF planar magnetron and ECR-hollow cathode reactive sputter deposition methods. Suitable plasma parameters have been identified to deposit CLD systems comprising a $335 \mu \mathrm{m}$ alumina layer on a $25 \mu \mathrm{m}$ layer of a silicone elastomer and a $40 \mu \mathrm{m}$ alumina layer with Young's modulus of $220 \mathrm{GPa}$ on a commercial acrylic damping film.

Beam specimens treated with plasma CLD were tested over a broad range of frequencies and temperatures using two different test methods. Maximum loss factors around 0.03 were measured; this is typically 50 per cent more than could be achieved with a traditional CLD coating of the same weight.
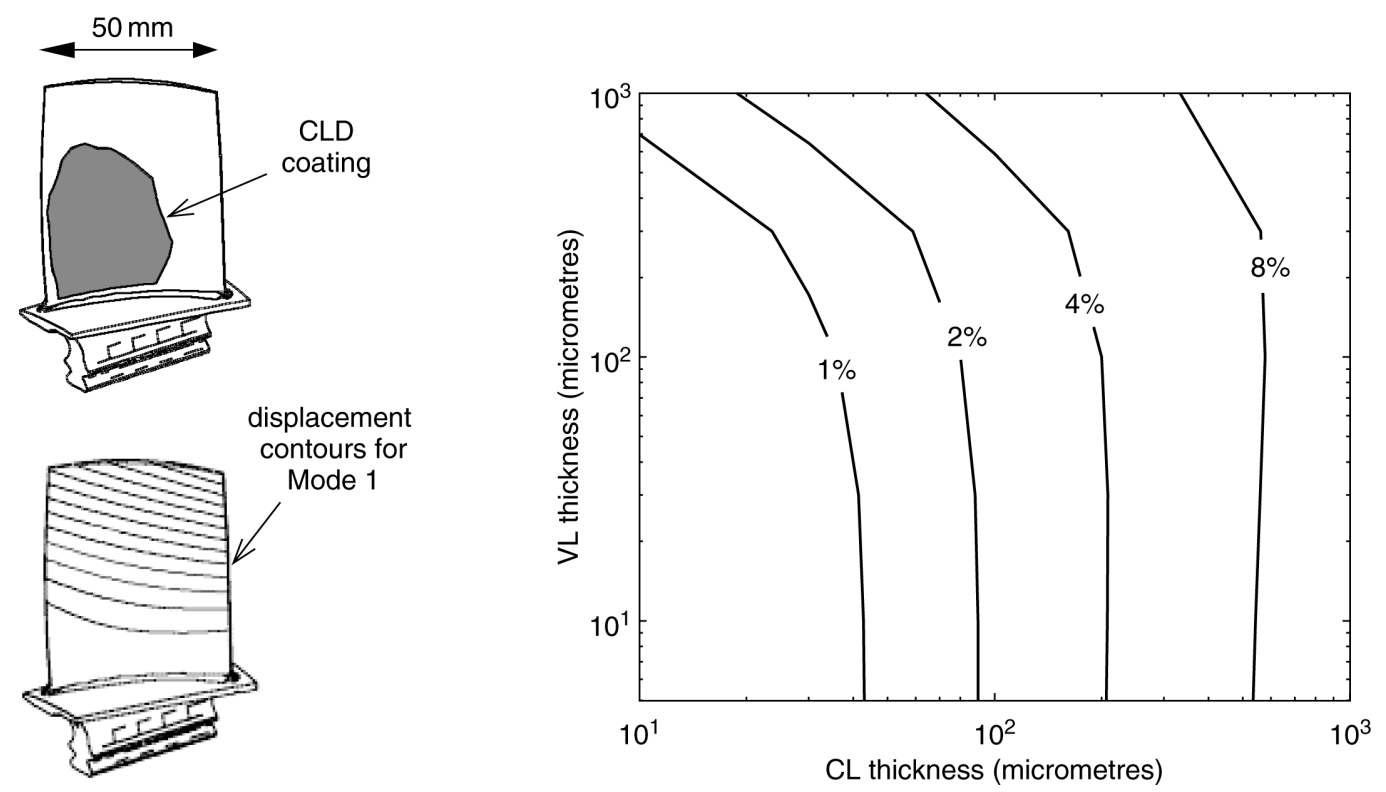

Fig. 11 Maximum loss factors of a compressor blade partially coated with plasma CLD (contours represent damping) 

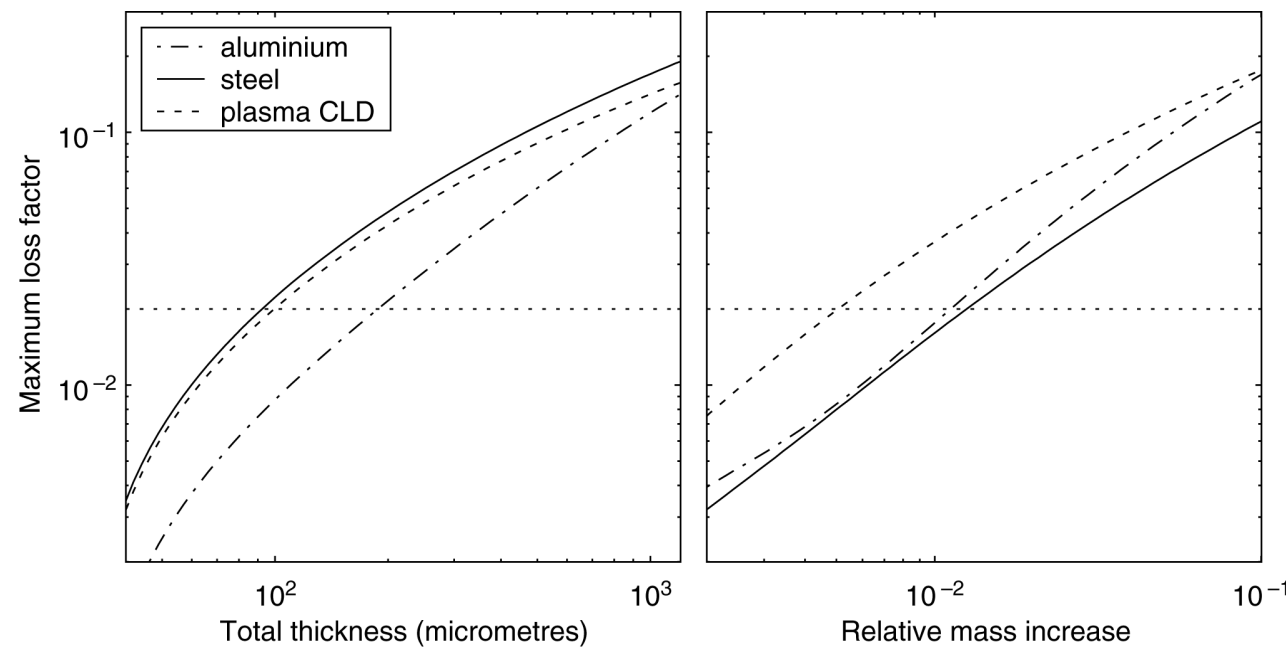

Fig. 12 Comparison of maximum loss factors of the compressor blade partially coated with various treatments when the viscoelastic layer thickness is $30 \mu \mathrm{m}$

Numerical techniques for predicting the performance of CLD systems were evaluated. The simple approach of Marsh and Hale was shown to give more accurate results for the peak loss factor than the common MSE method. A comparison was made between measured and predicted results over different temperatures and several vibration modes. This study indicated that the deposition process for the ceramic layer may reduce the loss factor of some materials, in this case the damping tape ISD112.

Future work should address plasma-deposited viscoelastic layers that have low modulus and high damping. This study will require the development of characterization methods for thin viscoelastic layers, in particular nanoindentation techniques.

A second area requiring improvement is in the growth rate of coatings. In this work, growth rates up to $13 \mu \mathrm{m} / \mathrm{h}$ were achieved for alumina. For increased use, this would have to be increased dramatically to deposit coatings $100+\mu \mathrm{m}$ in thickness. Although higher rates are achievable (electron beam evaporation, for example, yields alumina coatings with rates up to $1200 \mu \mathrm{m} / \mathrm{h}$ [31]), ways of increasing the power density on the target material without degrading damping must be found.

\section{ACKNOWLEDGEMENTS}

This work was supported by the Engineering and Physical Sciences Research Council (EPSRC), UK, under Grant GR/M36144/01. Useful discussions with Dr R. Short of the Department of Engineering Materials at the University of Sheffield are gratefully acknowledged.

\section{REFERENCES}

1 Nashif, A. D., Jones, D. I. and Henderson, J. P. Vibration Damping, 1985 (John Wiley, New York).

2 Garibaldi, L. and Onah, H. N. Viscoelastic Material Damping Technology, 1996 (Becchis Osiride, Torino, Italy).

3 Biggerstaff, J. M. and Kosmatka, J. B. Shear measurements of viscoelastic materials embedded in composite plates. In SPIE Smart Structures and Materials Conference on Passive Damping and Isolation, Newport Beach, California, March 1999, Vol. 3672, pp. 82-92.

4 Biederman, H. and Osada, Y. Plasma Polymerisation Processes, 1992 (Elsevier, Amsterdam, The Netherlands).

5 Aita, C. R. Tailored ceramic film growth at low temperature by reactive sputter deposition. Critical Rev. Solid State Mater. Sci., 1998, 23, 205-274.

6 Chatterjee, S. and Chandrashekhar, S. Deposition methods, properties and metal cutting applications of ceramic films. Key Engng Mater., 1996, 114, 67-104.

7 Movchan, B. A., Malashenko, I. S., Yakovchuk, K. Yu., Rybnikov, A. I. and Tchizhik, A. A. Two and three layer coatings produced by deposition in vacuum for gas turbine blade protection. Surf. Coating Technol., 1994, 67, 55-64.

8 Cueff, R., Baud, G., Benmalek, M., Besse, J. P., Butruille, J. R., Dunlop, H. M. and Jacquet, M. Characterisation and adhesion study of thin alumina coatings sputtered on PET. Thin Solid Films, 1995, 270, 230-236.

9 Bodino, F., Baud, G., Benmalek, M., Besse, J. P., Dunlop, H. M. and Jacquet, M. Alumina coating on polyethylene terephthalate. Thin Solid Films, 1994, 241, 21-24.

10 Marsh, E. R. and Hale, L. C. Damping of flexural waves with embedded viscoelastic materials. Trans. ASME, J. Appl. Mechanics, 1998, 120, 188-193.

11 Ross, C. A. Properties of radio-frequency-sputtered alumina films on flat and grooved substrates. J. Vacuum Sci. Technol. A, 1996, 14, 2511-2516.

12 Plunkett, R. and Lee, C. T. Length optimisation for constrained viscoelastic layer damping. J. Acoust. Soc. Am., 1970, 48, 150-161. 
13 Demoret, K. J. and Torvik, P. J. Optimal length of constrained layers on a substrate with linearly varying strain. In Proceedings of ASME Design Engineering Technical Conference, Boston, Massachusetts, Vol. 3(C), 1995, pp. 719-726.

14 Rongong, J. A. Shear and extensional behaviour of passive and active constrained layer damping. PhD thesis, University of Sheffield, 2002.

15 Morinaka, A. and Asano, Y. Residual stress and thermal expansion coefficient of plasma polymerised films. J. Appl. Polym. Sci., 1982, 27, 2139-2150.

16 Rodriguez, F. Principles of Polymer Systems, 1983 (McGraw-Hill, Singapore).

17 Wrobel, A. M. Mechanism of plasma polymerisation of $\mathrm{N}$ silyl-substituted cyclodisilazane; structure and properties of polymer film. Plasma Chem. Plasma Process, 1987, 7, 429450 .

18 Materials Information Sheet: silicone elastomers-Sylgard 182 and 184, Dow Corning Corporation, 1998.

19 Technical Data Sheets: viscoleastic damping polymers 110, 112 and 130, 3M, Bonding Systems Division, St Paul, Minnesota, 1999.

20 Zhu, S., Wang, F., Lou, H. and Wu, W. Reactive sputter deposition of alumina films on superalloys and their hightemperature corrosion resistance. Surf. Coatings Technol., 1995, 71 (1), 9-15.

21 Ono, T., Takahashi, C. and Matsuo, S. Electron cyclotron resonance plasma deposition technique using raw material supply by sputtering. Jap. J. Appl. Physics, 1984, 23, L534L536.

22 Oliver, W. C. and Pharr, G. M. An improved technique for determining hardness and elastic modulus using load and displacement sensing indentation experiments. J. Mater. Res., 1992, 7, 1564-1583.
23 Golla, D. F. and Hughes, P. C. Dynamics of viscoelastic structures - a time-domain finite element formulation. Trans. ASME, J. Appl. Mechanics, 1985, 52, 897-906.

24 Slater, J. C., Belvin, W. K. and Inman, D. J. A survey of modern methods for modelling frequency dependent damping in finite element models. In Proceedings of the 11th International Modal Analysis Conference, Kissimmee, Florida, 1993, pp. 1508-1512.

25 Wilson, C. J., Carnevali, P., Morris, R. B. and Tsuji, Y. Viscoelastic damping calculations using a p-type finite element code. Trans. ASME, J. Appl. Mechanics, 1992, 59, 696-699.

26 Johnson, C. D. and Kienholz, D. A. Finite element prediction of damping in structures with constrained viscoelastic layers, Am. Inst. Aeronaut. Astronaut. J., 1982, 20, 1284-1290.

27 Rongong, J. A. Damped joints in thin plate structures. In Proceedings of ISMA 25 on Noise and Vibration in Engineering, Leuven, Belgium, 2000, pp. 817-824.

28 Miles, R. N. and Reinhall, P. G. Modelling of a hybrid constraining layer piezoceramic approach in active damping. Trans. ASME, J. Vibr. Acoust., 1997, 119, 277287.

29 Sanliturk, K. Y. Finite Element Software FINES, Version 2.1C, 1999 (Istanbul Technical University, Istanbul, Turkey).

30 Ross, D., Ungar, E. E. and Kerwin, E. M. Damping of plate structural vibrations by means of viscoelastic laminae. In Structural Damping (Ed. J. Ruzicka), 1960 (Pergamon Press, New York).

31 Schiller, S., Goedicke, K. and Metzner, C. Plasma-activated high-rate electron-beam evaporation for coating metal strips. Mater. Sci. Engng A, 1993, 163, 149-156. 